\title{
ORBITS OF GALACTIC GLOBULAR CLUSTERS FROM SCHMIDT PLATE ASTROMETRY
}

\author{
M. ODENKIRCHEN ${ }^{1}$, R.-D. SCHOLZ ${ }^{2}$ and M.J. IRWIN ${ }^{3}$ \\ ${ }^{1}$ Sternwarte der Universität Bonn, Auf dem Hügel 71, D-53121 Bonn, Germany \\ ${ }^{2}$ WIP-Projekt Astrometrie bei der Universität Potsdam, An der Sternwarte 16, \\ D-14482 Potsdam, Germany \\ ${ }^{3}$ Royal Greenwich Observatory, Madingley Road, Cambridge CB3 OEZ, U.K.
}

\begin{abstract}
We present results from orbit integrations for the globular clusters $M 3$ and $M 92$. Absolute proper motions recently measured from Tautenburg Schmidt plates and a three-component mass model for the Galaxy have been used to derive the galactic orbits of these clusters. Orbital parameters and the influence of observational uncertainties on the determination of the orbits are discussed.
\end{abstract}

\section{Introduction}

Our present knowledge about the kinematics and dynamics of the system of galactic globular clusters is still very restricted. Because of an almost total lack of reliable absolute proper motion data, investigations had to be based on radial velocities alone. The crucial point for kinematical studies is that the clusters' proper motions must be absolute, i.e. measured with respect to an inertial frame of reference, to a high degree of accuracy. Only during the last decade have successful attempts been made to solve this problem by deriving cluster proper motions with respect to an extragalactic reference system (Brosche et al. 1985, 1991; Tucholke 1992; Geffert et al. 1993). Recently, the use of Schmidt plates has brought considerable progress to globular cluster astrometry. Schmidt plates allow for the first time the measurement of the proper motion of cluster stars directly against a very large number of background galaxies. This has been demonstrated with plates from the Tautenburg Schmidt telescope in the work of Scholz \& Irwin (1993). They determined absolute proper motions of the globular clusters M 3 and $M 92$ with an overall accuracy of 0.02 to 0.07 arcsec/100a. Therefore, these two clusters are now the best candidates for a study of orbital motion in the galactic halo.

\section{Initial Values and Galactic Model}

The orbit of a globular cluster in the Galaxy is uniquely determined by a complete set of kinematic data at one epoch and by the forces acting on the cluster along the orbit.

In order to derive the present space motions of $M 3$ and $M 92$, we have combined the absolute proper motions of Scholz \& Irwin (1993) with radial velocities from Gunn \& Griffin (1979) and Webbink (1981) and with distances from Paez et al. (1990) and Sandage (1970, 1983). These data are listed in Table 1 together with error estimates. For reduction of positions and space 
motions to a galactocentric frame, assumptions on the Sun's location and motion in the Galaxy must be made. Adopting a distance Sun-galactic center of $8.0 \pm 0.5 \mathrm{kpc}$, a rotational velocity for the LSR of $225 \pm 20 \mathrm{~km} / \mathrm{s}$ and the standard solar motion as given e.g. in Johnson \& Soderblom (1987), we obtain the values of Table 2 . They describe in rectangular galactocentric components the present kinematic state of the two clusters and serve as initial values for the integration of the equations of motion.

In accordance with earlier papers (Brosche et al. 1991; Odenkirchen \& Brosche 1992; Geffert et al. 1993), the forces acting on the cluster are modelled by the galactic gravitational potential of Allen \& Martos (1986). This potential was designed especially for the purpose of orbit integration. It is generated by three mass components. Since it is stationary and axisymmetric, every orbit has two integrals of motion: the specific total energy $E$ and the specific angular component $\mathbf{J}_{2}$.

\section{The Orbit of $\mathbf{M 3}$}

We have backward integrated the equations of motion of the cluster over a time interval of 10 Gyr. Characteristic values of the resulting orbit are given in Table 3. Part of the orbit is also illustrated in Figs. 1 and 2 by projections into the meridional plane (spanned by the cylindrical coordinates $p$ and $z$ ) and the galactic plane. Figure 1 also contains the boundary curve which confines the meridional orbit due to the fixed values of $\mathrm{E}$ and $\mathrm{J}_{z}$. In fact, we find that the cluster remains within a much smaller area than given by this curve with distances between 5.1 and $12.3 \mathrm{kpc}$ from the galactic centre and up to $12 \mathrm{kpc}$ from the galactic plane. The envelope has the shape of a bent rectangular box. It touches the boundary curve only with its corners and is filled by the meridional orbit similar to a Lissajous figure. Thus the orbit belongs to the familiar type of box orbits and the existence of a third integral of motion is implied.

The volume filled by the orbit in 3-dimensional space has the shape of a torus of thickness $6.5 \mathrm{kpc}$. In the projection of Fig. $2 \mathrm{M} 3$ 's prograde motion consists of rapidly rotating ellipses of oscillating eccentricity. The cluster performs 33.6 revolutions around the galactic center during $10 \mathrm{Gyr}$, thus an average period of revolution of $\approx 0.3 \mathrm{Gyr}$ follows. The orbit is highly inclined to the galactic plane at an almost constant angle of $69^{\circ}$. This underlines its halo character.

We have also investigated the frequency distribution of some orbital parameters along the orbit. A clear trend towards large distance and low velocity is found (cf. time averages in Table 3). For example, the rotational velocity component $\Theta$ remains below $100 \mathrm{~km} / \mathrm{s}$ for more than $80 \%$ of the time. The present values of the orbital parameters nicely agree with these results, because they fall into ranges of high frequency.

\section{The Orbit of $M 92$}

Again we have calculated the orbit for $10 \mathrm{Gyr}$ back from present. Results are given in terms of parameter values (Table 3) and projections into the meridional plane (Fig. 3) and the galactic plane (Fig. 4). For M 92 we find an extremely small positive value of the angular momentum component $J_{z}$. This indicates very slow and retrograde rotation around the Galaxy's symmetry axis and allows for a rather peculiar type of orbit. According to the boundary curve in Fig. 3 defined by the integrals $\mathbf{E}$ and $\mathrm{J}_{z}$, the cluster could approach the galactic centre very closely. In 


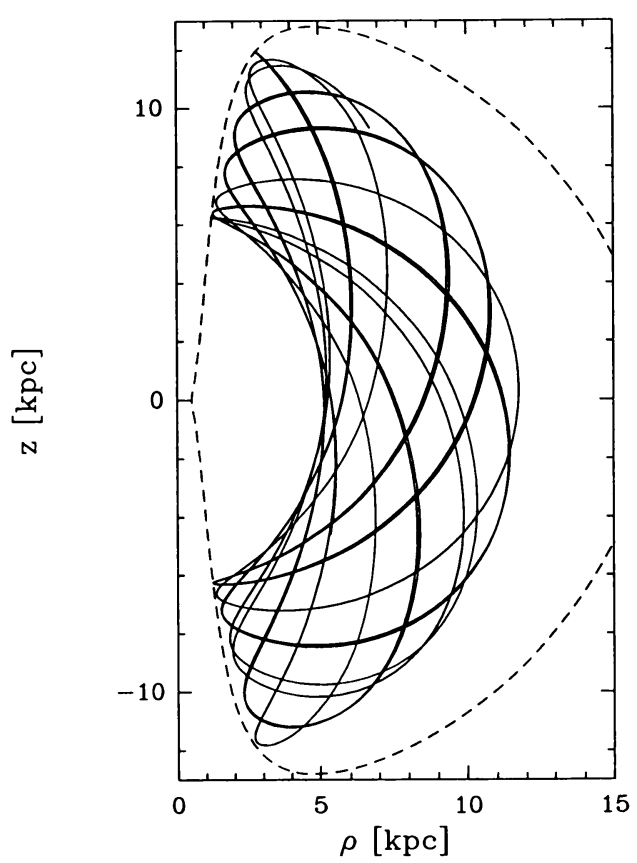

Figure 1. Orbit of $M 3$ in the time interval $[-3,0]$ Gyr, projected into the meridional plane. The dashed curve marks the boundary of orbital motion given by the values of the two integrals of motion.

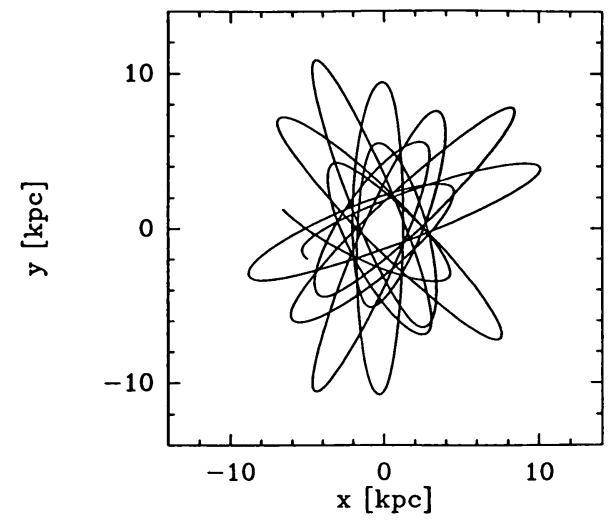

Figure 2. Orbit of $M 3$ in the time interval $[-3,0]$ Gyr, projected into the galactic plane. Approximately 10 prograde revolutions around the galactic center are performed during this time.

fact, the orbit keeps a distance of at least $1.4 \mathrm{kpc}$ from the centre. Very remarkably, it extends up to $5.8 \mathrm{kpc}$ into the northern part, but only $1.8 \mathrm{kpc}$ into the southern part of the Galaxy. Moreover, the values of orbital parameters $\rho$ and $z$ are strongly correlated. Consequently the envelope of the meridional orbit is asymmetric with respect to the galactic plane. This implies that the orbit is different from the usual box type. We characterize it by the term 'tube orbit'. A clear distinction between both types can be made by looking at the structure in phase space (see Fig. 6). While box orbits form a large island around the central fixed point in the Poincare section, tube orbits belong to different islands around one of the fixed points in the upper or lower part of the diagram.

Viewed from the North Galactic Pole (Fig. 4) the cluster appears to move on extremely elongated ellipses, which have almost constant eccentricity and rotate also in retrograde sense. Thus in 3-dimensional space the orbit is similar to the path of a Foucault pendulum. During 10 Gyr, 35 revolutions of the cluster around the galactic centre are counted. This yields a mean period of revolution of 0.28 Gyr. The velocity of the cluster varies between $6 \mathrm{~km} / \mathrm{s}$ near apogalacticon and $390 \mathrm{~km} / \mathrm{s}$ near perigalacticon. Therefore we find that large distances from the centre are much more likely along the orbit than small distances. Consequently the cluster spends more than $80 \%$ of the time north of the galactic plane. The rotational velocity component $\Theta$ is as small as $16 \mathrm{~km} / \mathrm{s}$ in absolute value on average and most likely even lower. Again, these tendencies fit in very well with the observed present values. 


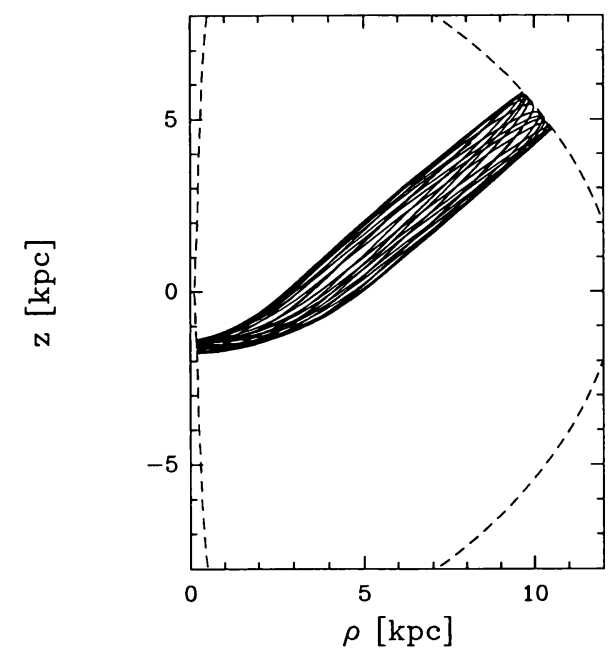

Figure 3. Orbit of $\mathrm{M} 92$ in the time interval $[-2,0]$ Gyr, projected into the meridional plane. The dashed curve marks the boundary of orbital motion given by the values of the two integrals of motion.

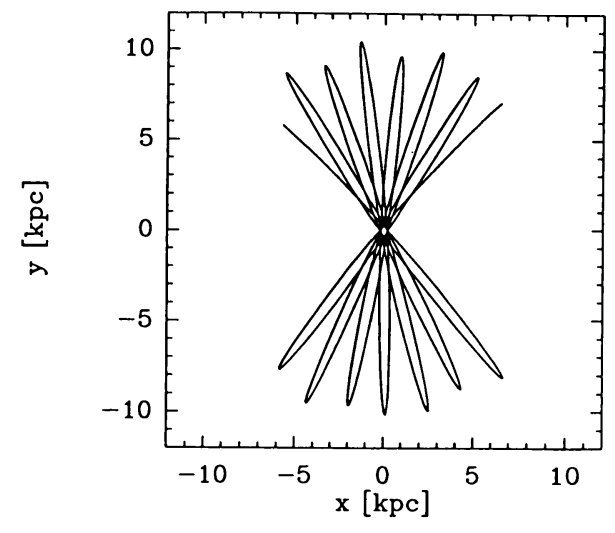

Figure 4. Orbit of $\mathrm{M} 92$ in the time interval $[-2,0]$ Gyr, projected into the galactic plane. 7.25 retrograde revolutions around the galactic center can be recognised.

\section{Influence of Observational Errors}

The influence of observational errors on the given orbits were investigated by calculating for each cluster four test orbits with changed initial values. The variations in the initial values were derived from the errors given in Table 1 and Section 2.

For $M 3$ the resulting orbits are all of box type and their envelope remains of similar shape and size. Apogalactic distance and maximum distance from the galactic plane are almost the same for all orbits, whereas perigalactic distance varies by about $\pm 20 \%$. This yields eccentricity values between 0.3 and 0.5 . According to an analysis of the uncertainty of the integrals of motion, the sign of $J_{z}$ and therefore the sense of rotation is well determined.

In the case of M 92 three of the four orbits with changed initial values are again tube orbits with similar size and the same north-south-asymmetry as described in Section 4. Distances to galactic centre and galactic plane vary by about $\pm 10 \%$, eccentricity ranges from 0.77 to 0.81 . However, there remains some uncertainty about the sign of $J_{z}$ and hence about the retrograde sense of rotation, because the value of $J_{z}$ is so close to zero. The fourth test orbit deviates largely from the former ones over time intervals of several Gyr. Figure 5 shows that it runs erratically through the whole interior of the zero velocity curve. Indeed the Poincare section (Fig. 6) reveals that this is a stochastic orbit. We conclude that for M 92 a transit from tube orbit to stochastic orbit may be possible at the given level of uncertainty in the determination of initial values. 


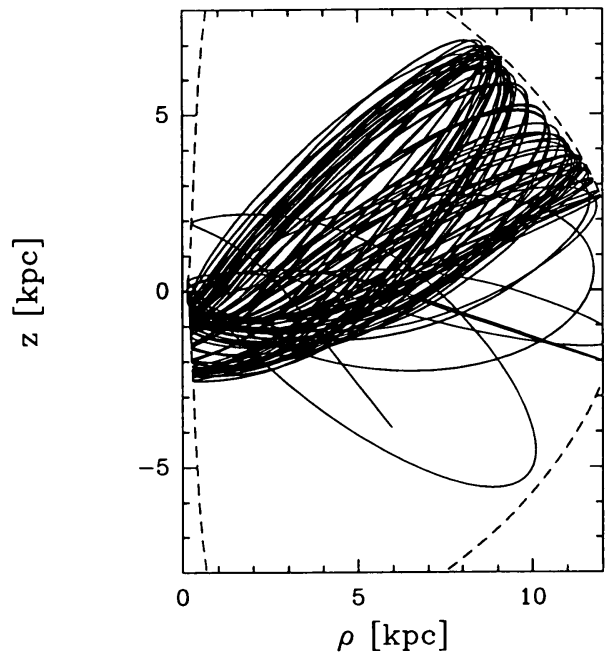

Figure 5. Meridional projection of a stochastic orbit corresponding to changed initial values of $\mathbf{M}$ 92. Here the time interval is $[-10,0]$ Gyr. Again the dashed line marks the zero velocity boundary.

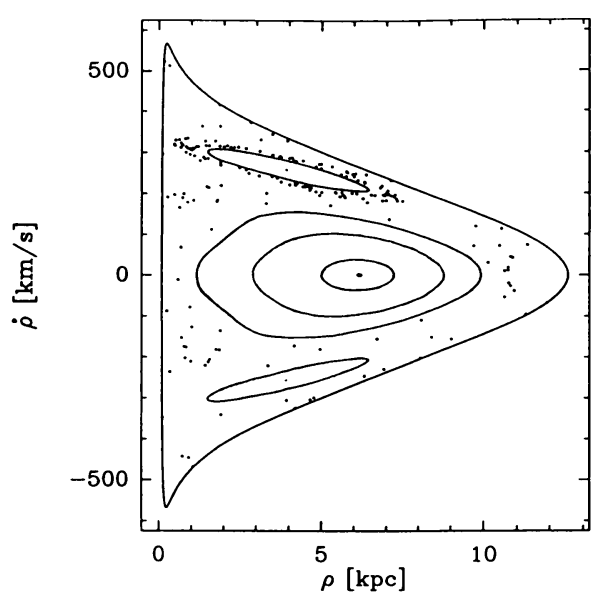

Figure 6. Poincare section of the stochastic orbit of Fig. 5 (dots) in phase space. Solid lines within the triangle indicate islands of regular motion. The central dots within these islands correspond to simple periodic orbits in the meridional plane.

Table 1. Observational cluster data

\begin{tabular}{|c|c|c|c|c|c|c|c|c|}
\hline \multirow[t]{2}{*}{ Object } & \multicolumn{2}{|c|}{$\alpha(1950)$} & \multicolumn{2}{|c|}{$\delta(\mathbf{1 9 5 0})$} & \multirow{2}{*}{$\begin{array}{c}D \\
{[\mathbf{k p c}]}\end{array}$} & \multirow{2}{*}{$\begin{array}{c}v_{r} \\
{[\mathbf{k m} / \mathbf{s}]}\end{array}$} & $\mu_{\alpha} \cos \delta$ & \multirow{2}{*}{${ }_{\text {[00a }]}^{\mu_{\delta}}$} \\
\hline & $\mathbf{h}$ & $\mathbf{m}$ & $\bullet$ &, & & & [arcsec/100a] & \\
\hline M 3 & 13 & 39.9 & 28 & 38 & $9.5 \pm 1.0$ & $-146.9 \pm 0.5$ & $-0.31 \pm 0.02$ & $-0.23 \pm 0.04$ \\
\hline M 92 & 17 & 15.6 & 43 & 11 & $7.6 \pm 0.7$ & $-120.5 \pm 1.7$ & $-0.44 \pm 0.07$ & $+0.11 \pm 0.04$ \\
\hline
\end{tabular}

Table 2. Derived galactocentric position and velocity

\begin{tabular}{l|lll|lll}
\hline Object & $x$ & $y$ & $z$ & $U$ & $\begin{array}{l}V \\
{[\mathrm{~km} / \mathrm{s}]}\end{array}$ & $W$ \\
\hline \hline M 3 & $-6.6 \pm 0.5$ & $1.3 \pm 0.1$ & $9.3 \pm 1.0$ & $-53 \pm 14$ & $54 \pm 31$ & $-108 \pm 4$ \\
M 92 & $-5.7 \pm 0.5$ & $5.8 \pm 0.5$ & $4.3 \pm 0.4$ & $-80 \pm 15$ & $70 \pm 26$ & $72 \pm 24$ \\
\hline
\end{tabular}


Table 3. Orbital parameters during $10 \mathrm{Gyr}$

Integrals of motion

\begin{tabular}{l|cc}
\hline & $\begin{array}{c}J_{z} \\
{[\mathrm{kpc}-\mathbf{k m} / \mathbf{s}]}\end{array}$ & $\begin{array}{c}E \\
{\left[\mathrm{~km}^{2} / \mathbf{s}^{2}\right]}\end{array}$ \\
\hline \hline M 3 & -290 & -116900 \\
M 92 & 65 & -128500 \\
\hline
\end{tabular}

Geometry of the orbit

\begin{tabular}{|c|c|c|c|c|c|c|c|}
\hline & $\boldsymbol{R}_{\min }$ & $\boldsymbol{R}_{\max }$ & $\overline{\boldsymbol{R}}$ & $R(t=0)$ & $e$ & $z_{\min }$ & $z_{\max }$ \\
\hline $\begin{array}{l}\text { M } 3 \\
\text { M } 92\end{array}$ & $\begin{array}{l}5.1 \\
1.4\end{array}$ & $\begin{array}{l}12.3 \\
11.6\end{array}$ & $\begin{array}{l}9.4 \\
8.0\end{array}$ & $\begin{array}{c}11.5 \\
9.2\end{array}$ & $\begin{array}{l}0.39 \\
0.78\end{array}$ & $\begin{array}{c}-12.0 \\
-1.8\end{array}$ & $\begin{array}{c}12.0 \\
5.8\end{array}$ \\
\hline
\end{tabular}

Absolute value of velocity

\begin{tabular}{l|cccc}
\hline & $v_{\text {min }}$ & $v_{\text {max }}$ & $v$ & $v(t=0)$ \\
& & & {$[\mathrm{km} / \mathrm{s}]$} & \\
\hline \hline M 3 & 104 & 331 & 186 & 132 \\
M 92 & 6 & 391 & 167 & 128 \\
\hline
\end{tabular}

Rotation around z-axis

\begin{tabular}{|c|c|c|c|c|c|c|}
\hline & $\Theta_{\min }$ & $\Theta_{\max }$ & $\Theta^{-}$ & $\Theta(t=0)$ & $\begin{array}{c}\bar{T} \\
{[\mathbf{G y r}]} \\
\end{array}$ & $n_{U}$ \\
\hline $\begin{array}{l}\text { M } 3 \\
\text { M } 92\end{array}$ & $\begin{array}{c}25 \\
-387\end{array}$ & $\begin{array}{c}237 \\
-6\end{array}$ & $\begin{array}{c}66 \\
-16\end{array}$ & $\begin{array}{l}43 \\
-8\end{array}$ & $\begin{array}{l}0.30 \\
0.28\end{array}$ & $\begin{array}{l}33.6 \\
35.2\end{array}$ \\
\hline
\end{tabular}

Definition of symbols used in Tables.

D distance from object to observer

$v_{r} \quad$ radial velocity

$x, y, z \quad$ galactocentric space coordinates in a right-handed cartesian coordinate system with $z$ pointing to North Galactic Pole, $x$ away from the Sun and $y$ in direction of galactic rotation at the Sun.

$U, V, W \quad$ corresponding velocity components

$\boldsymbol{R} \quad$ Distance from the galactic centre

$p \quad$ projected distance in galactic plane

min,max as index means minimum and maximum during $10 \mathrm{Gyr}$

- above letter means time average in $10 \mathrm{Gyr}$

e eccentricity defined as $\left(R_{\max }-R_{\min }\right) /\left(R_{\max }+R_{\text {min }}\right)$

$v \quad$ absolute value of velocity 
$\Theta$ component of velocity vector in direction of galactic rotation at the position of the cluster $T \quad$ period of one revolution around $z$-axis

$n_{U} \quad$ number of revolutions in $10 \mathrm{Gyr}$

$J_{z} \quad z$-component of specific angular momentum vector

$E \quad$ specific total orbital energy

\section{References}

Allen, C. and Martos, M.A., 1986. Rev. Mex. Astron. Astrofis., 13, 137.

Brosche, P., Geffert, M., Klemola, A.R. and Ninkovic, S., 1985. Astron. J., 90, 2033.

Brosche, P., Tucholke, H.-J., Klemola, A.R., Ninkovic, S., Geffert, M. and Doerenkamp, P., 1991. Astron. J., 102, 2022.

Geffert, M., Colin, J., LeCampion, J.F. and Odenkirchen, M., 1993. Astron. J., 106, 168.

Gunn, J.E. and Griffin, R.F., 1979. Astron. J., 84, 752.

Johnson, D.R.H. and Soderblom, D.R., 1987. Astron. J., 93, 864.

Odenkirchen, M. and Brosche, P., Astron. Nachr., 313, 69.

Paez, E., Straniero, O. and Martinez, R.C., 1990. Astron. \& Astrophys. Suppl., 84, 481.

Sandage, A., 1970. Astrophys. J., 162, 841.

Sandage, A., 1983. Astron. J., 88, 1159.

Scholz, R.-D. and Irwin, M.J., 1993. IAU Symp., 156, 249.

Tucholke, H.-J., 1992. Astron. \& Astrophys. Suppl., 93, 311.

Webbink, R.F., 1981. Astrophys. J. Suppl., 45, 259. 